Maja Pawlowska

Université de Wrocław

\title{
ENSEIGNEMENT DE LA LITTÉRATURE FRANÇAISE ANCIENNE : SOUVENIRS ET TÉMOIGNAGE
}

Faire un bilan des quarante dernières années d'enseignement de la littérature française ancienne en Pologne relève de la gageure. Une telle entreprise, pour être complète, nécessiterait un travail collectif des enseignants, sociologues et historiens. Ainsi, plus modestement, sans ambitionner de dresser un panorama exhaustif des changements survenus dans tous les départements de français en Pologne, j'aimerais offrir un témoignage personnel qui, bien sûr, ne porte que sur un seul établissement, l'Institut de Philologie Romane de l'Université de Wrocław, mais celui-ci peut être considéré comme un modèle représentatif des transformations. J'ai eu la chance de pouvoir observer l'enseignement de la littérature française ancienne (englobant la période du Moyen Âge à la fin de l'Ancien Régime) dans cet Institut depuis presque quatre décennies, d'abord comme étudiante, ensuite comme enseignante. La ponctualité de l'exemple est ainsi contrebalancée par une vue de longue haleine et par une double perspective. Ces deux composantes incitent tout naturellement à réfléchir sur le sens des études de littérature étrangère — dans mon cas, la littérature française ancienne — dans l'enseignement universitaire contemporain.

En Pologne, la chute du régime communiste en 1989 a entraîné des répercussions profondes et variées non seulement dans la vie politique, mais aussi dans tous les aspects de la vie des Polonais. Son impact direct sur les composantes essentielles de l'enseignement, c'est-à-dire sur l'étudiant, l'enseignant et le programme d'études, est incontestable. Je vais structurer ma réflexion autour de ces trois éléments, afin de mettre en relief leurs intrications et d'essayer d'en dégager 
une réponse plausible à la question de la place que doit ou peut occuper la littérature ancienne française dans les études universitaires.

L'Institut de Philologie Romane ${ }^{1}$ de l'Université de Wrocław a été créé en 1973 à la suite de la transformation et du développement d'une chaire de philologie romane préexistante. Dès le début, on y a enseigné la littérature française, qui demeure un des trois piliers du programme de formation des étudiants en philologie française $^{2}$. Si l'on jette un regard rétrospectif sur son fonctionnement, une nette division en deux époques distinctes apparait, et elle s'articule autour de la date de 1989. Les changements les plus frappants se présentent dans le profil des étudiants, mais restent en corrélation étroite avec les deux autres composantes mentionnées.

Pour mieux comprendre ce qui a pu changer au cours des quarante dernières années, il faudrait commencer par dresser un état des conditions de l'enseignement de la littérature française ancienne en Pologne dans les années soixantedix et quatre-vingt. Pendant cette période, avoir un diplôme d'études supérieures n'impliquait pas que l'on reçoive un salaire plus élevé que celui d'un ouvrier. Ce dernier était souvent mieux rémunéré qu'un professeur de français. En outre, les différences de salaires entre personnes diplômées n'étaient pas notables. De ce fait, les facteurs économiques intervenaient peu dans le choix des études. Les motivations qui poussaient les jeunes à préférer les études romanes relevaient davantage de choix personnels. Ils devaient pourtant être bien réfléchis, car si toutes les écoles supérieures polonaises étaient publiques et gratuites, le nombre de places en première année était fixe et limité, et il y avait une limite d'âge d'admission aux études supérieures ${ }^{3}$. C'est ainsi que seuls dix pour cent des jeunes de 18 à 24 ans étudiaient dans un établissement supérieur ${ }^{4}$.

Pour être admis aux études françaises, les candidats devaient non seulement réussir leur baccalauréat de français et de polonais, mais aussi passer un examen d'entrée. C'était un concours qui comprenait des épreuves écrites et orales pour vérifier la connaissance de la langue française, et un examen oral de grammaire et de littérature polonaises. En général, le nombre de candidats était deux à trois fois plus élevé que le nombre des places disponibles : la sélection permettait donc d'imposer un niveau élevé de maîtrise du français, équivalant au B2 de l'actuel cadre européen commun de référence pour les langues. De même, les connaissances en littérature et grammaire de la langue maternelle devaient nécessairement être élevées, à un niveau dépassant celui du programme des lycées. Pour

${ }^{1}$ Cette appellation correspond dans les universités françaises à celle du département d'études françaises et/ou espagnoles et/ou italiennes.

${ }^{2}$ Les deux autres sont la linguistique et l'apprentissage de la langue française.

${ }^{3}$ Cette limite était fixée à 28 ans. Il y avait cependant des filières d'études supérieures dont l'accès était réservé aux personnes ayant dépassé cet âge, professionnellement actives. Mais vu leur spécificité, elles n'entrent pas dans le champ de la présente réflexion.

${ }^{4}$ Cf. <http://www.nauka.gov.pl/g2/oryginal/2013_07/0695136d37bd577c8ab03acc5c59a1f6. pdf> [consulté le 30.10.2017]. 
réussir le concours, il était donc impératif de consacrer beaucoup de temps à s'y préparer, d'être motivé, d'avoir un don pour l'apprentissage des langues étrangères et de l'intérêt pour les sciences humaines, littérature comprise.

En règle générale, les jeunes qui entamaient leurs études de philologie romane avaient un savoir rudimentaire sur la littérature française acquis au lycée et par leurs lectures personnelles. Les textes des grands classiques tels que Villon, Rabelais, Corneille, Molière, pour ne citer que les auteurs les plus lus, ayant été traduits en polonais au début du vingtième siècle, étaient accessibles dans les bibliothèques publiques. La politique éditoriale de l'époque favorisait d'ailleurs les traductions de textes classiques européens qui, tout en assurant une distraction de valeur et un épanouissement intellectuel incontestable, étaient " politiquement correctes ». Il faut souligner aussi que jusqu'en 1989, ce que l'on nomme « littérature de masse », « littérature populaire » ou " paralittérature » constituait une partie minime de l'offre éditoriale polonaise. L'objectif déclaré de cette dernière était de former et non de « flatter » les esprits des lecteurs. C'est ainsi que les candidats aux études philologiques avaient un goût littéraire formaté pour la " grande littérature ». Le côté négatif de cette situation, c'était le nombre relativement restreint de titres accessibles. L'offre éditoriale polonaise ne pouvait être complétée par des acquisitions originales à l'étranger, car le rideau de fer s'ouvrait difficilement, les voyages en France étaient très limités, et les prix mêmes des livres les mettaient hors de portée du voyageur polonais moyen ${ }^{5}$. En résultat de ce cloisonnement, les œuvres françaises non traduites en polonais étaient méconnues, exception faite des fragments de textes littéraires contenus dans les manuels d'apprentissage du français.

Pour compléter ce profil de l'étudiant en philologie romane de l'époque, quelques remarques concernant son éducation préalable s'imposent. La première concerne le choix du français comme langue étrangère étudiée. Après avoir terminé l'école primaire, l'élève pouvait poursuivre sa scolarité au lycée d'enseignement général qui durait quatre ans et se terminait par le baccalauréat. Parmi les matières enseignées, il y avait obligatoirement une langue occidentale : anglais, français ou allemand. La probabilité de pratiquer cette langue plus tard, dans la vie professionnelle, n'était pas élevée. La perspective de rencontrer des locuteurs natifs en voyage à l'étranger ou parlant leur langue en Pologne était peu probable. Le choix de la langue étrangère n'avait donc pas de fondements pratiques, il découlait de l'offre de l'établissement secondaire et des prédilections de l'élève. Avant le changement de régime, $50 \%$ des élèves apprenaient l'allemand comme langue étrangère occidentale, $37,5 \%$ l'anglais et $12,5 \%$ le français ${ }^{6}$.

Quant à la préparation littéraire, elle progressait régulièrement pendant toute la scolarité, les lectures littéraires faisant partie de l'enseignement de la langue

${ }^{5}$ Les différences de salaires en Pologne et en France étaient considérables.

${ }^{6}$ K. Wróblewska-Pawlak, I. Strachanowska, «Preferencje językowe młodzieży polskiej w okresie transformacji 1990-1999 », Studia Europejskie 1, 2000, pp. 13, 99-114, <http://www. ce.uw.edu.pl/pliki/pw/1-2000_Wroblewska-Pawlak.pdf > [consulté le 29.10.2017]. 
polonaise : langue et littératures étaient considérées comme indissociables. Le contenu des programmes de polonais (et d'autres matières) était élaboré par une commission ministérielle qui dressait une liste d'œuvres obligatoires et une anthologie complémentaire de titres, recommandés comme lectures individuelles pour les élèves ${ }^{7}$. Cette liste officielle répertoriait les œuvres majeures du patrimoine national et des textes faisant partie du patrimoine européen : textes fondateurs et mythiques, chansons de geste, chefs-d'œuvre classiques de renommée internationale. L'enseignement de la littérature se déroulait par ordre chronologique : on commençait par les mythes grecs pour arriver, en classe terminale, à la littérature contemporaine. Il n'y avait qu'un seul manuel de polonais, agrée par le ministère de l'éducation et utilisé dans tous les lycées. Ainsi, génération après génération, les Polonais possédaient en terminant leur éducation secondaire un tronc de références littéraires communes qui formait une culture partagée.

Les jeunes qui commençaient des études universitaires de français constituaient un groupe culturellement très homogène : tout le monde avait été formé sur la base des mêmes textes littéraires, les écarts étaient minimes. Le taux d'étudiants ayant effectué un séjour préalable en France, même de courte durée, ne dépassait pas dix pour cent des nouveaux effectifs. Dans cette situation, les étudiants, qui n'avaient pas de points de référence extérieurs, acceptaient le programme de littérature proposé et attendaient de la part du professeur qu'il leur explique un monde inconnu. Habitués dès l'enseignement primaire et secondaire à suivre un cours d'histoire des œuvres organisé selon leur chronologie, ils considéraient cette formule d'approche de la littérature comme une évidence. Le rôle du professeur dans le processus d'enseignement de la littérature était de premier plan : il ouvrait devant les étudiants le monde de la civilisation française, un monde autrement peu accessible. Lui seul détenait le savoir : il initiait les futurs romanistes à la culture étrangère en leur fournissant les interprétations qui donnaient du sens à ses textes. Dans ses explications, l'enseignant pouvait s'appuyer sur les connaissances préalables des étudiants, ce qui lui permettait de repérer facilement les lacunes de leurs compétences littéraires. Les cours de littérature s'appuyaient sur des manuels français, par exemple ceux de la collection Lagarde et Michard.

L'enseignement de la littérature française ancienne, qui ouvrait le parcours universitaire des étudiants, visait principalement à leur fournir une information essentielle sur l'histoire littéraire de la France et un résumé de ses œuvres majeures, afin de leur offrir une vue d'ensemble des siècles consécutifs. Le professeur devait endosser la fonction de guide, de maitre introduisant ses disciples dans un monde inconnu et les familiarisant avec une réalité culturelle ignorée jusqu'alors. En règle générale, les enseignants de philologie romane complétaient leurs études supérieures

${ }^{7}$ Natalia Paprocka, dans son article « Résonances de la littérature française à l'école polonaise dans les années 1946-1999» (Synergies Pologne 10, 2013, pp. 143-154, <https://gerflint.fr/Base/ Pologne10/paprocka.pdf $>$ ), décrit avec pertinence cette liste d'œuvres obligatoires à lire, en attribuant une attention particulière à la littérature française. 
en passant au moins une année dans une université francophone, et possédaient ainsi les compétences nécessaires pour engager le processus d'acculturation des étudiants.

Ce système d'enseignement, avec ses objectifs et sa spécificité, a subi de sérieuses mutations après 1989. Les principaux événements qui y ont contribué sont d'ordre politique (ouverture des frontières et adhésion de la Pologne à l'Union européenne), économique (convertibilité de la monnaie polonaise) et culturelles (apparition de nouvelles technologies de communication, diffusion intensive de la culture occidentale, principalement anglo-saxonne au sens large).

Le monde universitaire, le milieu des enseignants des philologies de langues occidentales tout particulièrement, a accueilli les transformations avec bienveillance. Il s'est pourtant avéré qu'on devait apprivoiser une réalité toute nouvelle. L'économie de marché ayant remplacé l'économie planifiée, une grande disparité des salaires et le chômage sont apparus. Désormais, le choix de la formation supérieure se traduisait en effets économiques tangibles. Sous l'impact de la refonte des assises sociales et économiques du pays, les études supérieures ont subi une redéfinition, et ce, dans deux directions.

La première se rapporte à la hiérarchisation des disciplines en termes de prestige, qui est devenue beaucoup plus prononcée qu'autrefois. La formation humaniste a commencé à perdre de la vitesse face aux autres types d'enseignement supérieur. La connaissance d'une langue étrangère a cessé d'être l'effet d'une prédilection de l'élève, un « art pour l'art », et est devenue un choix stratégique, susceptible d'orienter l'avenir tout entier de celui qui le fait. Vingt-cinq ans après le début des transformations, on peut dire avec certitude que le choix de la langue étrangère dans l'enseignement secondaire reflète grosso modo les avantages que sa connaissance offre sur le marché du travail en Pologne et/ou dans le monde. Ainsi, les proportions entre les langues étrangères enseignées au lycée ont changé totalement : l'anglais a imposé sa présence massive dans le système scolaire polonais à tous les niveaux, dès l'école maternelle. L'allemand a perdu sa priorité et n'a cessé de reculer, dans une moindre mesure toutefois que le français. Les statistiques l'indiquent clairement : pendant l'année scolaire 1997-1998, 40\% des écoliers apprenaient l'anglais, $30 \%$ l'allemand, $24 \%$ le russe, $5 \%$ le français et $1 \%$ d'autres langues. La progression de l'anglais est allée croissant, et en 2005, les proportions des langues étrangères choisies par les bacheliers ${ }^{8}$ étaient les suivantes : $76 \%$ pour l'anglais, $17 \%$

8 «L'examen de baccalauréat consiste en 2 parties : obligatoire et optionnelle. Dans la partie obligatoire, les élèves passent un examen oral et écrit de langue polonaise, d'une langue étrangère choisie, de mathématiques et, le cas échéant, de langue d'une minorité enseignée dans une école donnée. Dans la partie optionnelle, l'élève a le droit de choisir de 1 à 6 autres matières pour lesquelles il veut passer un examen. L'élève décide également s'il les passe au niveau de base ou au niveau avancé. Pour réussir au baccalauréat, il est nécessaire d'obtenir un total d'au moins $30 \%$ des points pour chaque matière examinée dans la partie obligatoire » $(<\mathrm{http}: / /$ www.migrant.info.pl/ types-decoles-en-pologne.html> [consulté le 29.10.2017]). 
pour l'allemand, pour $5,3 \%$ le russe, $1,5 \%$ pour le français et $0,2 \%$ pour les autres 9 . Cette tendance s'est perpétuée et, en 2017 , les chiffres respectifs sont : $91,7 \%$ pour l'anglais, $5,8 \%$ pour l'allemand $1,7 \%$ pour le russe et $0,8 \%$ pour le français et les autres langues ${ }^{10}$. Ainsi, le pourcentage de jeunes candidats potentiels aux études françaises a diminué fortement.

La seconde direction des mutations de l'enseignement supérieur survenues après 1989 c'est la démocratisation des études, les directives gouvernementales n'ayant cessé de favoriser l'accès des bacheliers à celles-ci. Ainsi, en 25 ans, le taux de la scolarisation supérieure a quintuplé, pour atteindre actuellement environ $50 \%$ des jeunes de 18 à 24 dans. À la section de français de l'Institut de Philologie Romane de l'Université de Wrocław, le nombre de places en première année, qui est fixé et limité chaque année, a triplé en $25 \mathrm{ans}^{11}$. L'admission aux études est tout de suite devenue moins élitaire, et le profil des candidats aux études de français a évolué. Pour une partie d'entre eux, le choix de ces études n'était plus dicté par un intérêt réel pour la langue et la civilisation françaises, mais par la relative facilité d'admission. La première année d'études est désormais souvent considérée comme un coup d'essai, et les abandons viennent rapidement.

En 1999, la Pologne a connu une profonde réforme de son système éducatif, tant du point de vue structurel qu'en matière de programmes. Le système de scolarité divisée en deux étapes - une école primaire obligatoire (de huit ans) suivie d'écoles d'enseignement secondaire, par exemple les lycées d'enseignement général (quatre ans) - a été remplacé par un système en trois étapes : école primaire obligatoire de six ans, puis collèges obligatoires de trois ans, puis écoles post-collège, dont les lycées d'enseignement général (trois ans). Les programmes scolaires ont été revus en conséquence. Le manuel unique a été remplacé par tout un éventail de manuels agréés par le ministère de l'éducation, l'enseignant pouvait désormais le choisir selon ses préférences ${ }^{12}$. L'objectif général de l'enseignement de la littérature a changé : on lui a assigné, outre sa fonction traditionnelle de transmission et d'interprétation des textes du patrimoine culturel national et européen «garants des valeurs sur lesquelles se fonde la société $»^{13}$, celle

${ }^{9}$ Cf. $<$ http://biqdata.wyborcza.pl/jezyki-obce $>$ [consulté le 29.10.2017].

${ }^{10} \mathrm{Cf}$. <http://www.cke.edu.pl/images/_EGZAMIN_MATURALNY_OD_2015/Informacje_o_wynikach/2017/20170630> [consulté le 29.10.2017].

${ }^{11}$ Le nombre des effectifs d'enseignants n'a pas suivi cette tendance, il a peu changé depuis 30 ans.

12 «Chaque enseignant a aussi la possibilité de déterminer individuellement son programme, en le soumettant à l'agrément du ministère, ce que peu font en réalité. La proportion entre cadre national obligatoire et développement particulier est de 70-30» (D. Ulma, « L'enseignement de la littérature à l'école primaire en Europe : quelques exemples de prescription institutionnelle », [dans :] École, médiations et réformes curriculaires : Perspectives internationales, R. Mallet (dir.), De Boeck, Bruxelles 2010, p. 53).

${ }^{13} \mathrm{Je}$ reprends ici la formule de Sylvaine Ahr (S. Ahr, L'enseignement de la littérature au collège, Éditions L’Harmattan, Paris 2006, p. 10). 
de former un esprit critique et de pourvoir l'élève des capacités intellectuelles nécessaires aux lectures individuelles réfléchies. Le précis historique, ou étude chronologique de la littérature, a été abandonné en faveur d'une approche thématique. Comme l'a résumé avec pertinence Dominique Ulma : «En Pologne, on souhaite surtout aborder via la littérature des thèmes universels, construire un être équilibré plus qu'un lecteur de littérature ${ }^{14}$. Ces nobles aspirations des réformateurs des programmes scolaires ont malheureusement apporté un effet secondaire défavorable : l'incapacité des jeunes de saisir la littérature européenne dans son aspect diachronique.

Les réformes du système éducatif ont aussi transformé l'enseignement supérieur. La structure des études françaises a été modifiée, le cycle unique de maitrise en 10 semestres étant désormais divisés en un premier cycle de licence de 6 semestres, et un deuxième cycle de master de 4 semestres. La répartition des cours de littérature française ancienne n'a toutefois pas été remaniée sensiblement : des deuxième et troisième semestres, ils ont été transposés aux premier et second semestres du premier cycle. De même, le nombre de semestres et d'heures consacrées aux cours d'histoire de la littérature française n'a pas changé ${ }^{15}$. La littérature médiévale est enseignée au premier semestre, celle du XVII ${ }^{\mathrm{e}}$ siècle au second, les semestres suivants portent sur les $\mathrm{XVIII}^{\mathrm{e}}$ et $\mathrm{XIX}^{\mathrm{e}}$ siècles, et les deux derniers semestres sont consacrés à la littérature du $\mathrm{XX}^{\mathrm{e}}$ siècle et contemporaine.

L'admission aux études ne se fait plus au moyen d'examens d'entrée, mais par concours, suivant les résultats du baccalauréat. La vérification des compétences linguistiques, littéraires et grammaticales a ainsi été dissociée des établissements universitaires. L'entretien avec les candidats a été remplacé par des procédures impersonnelles.

Quels sont les résultats de ces changements ? Le profil de l'étudiant commençant des études de français a perdu de son homogénéité. Le professeur de littérature qui donne les cours aux débutants est confronté chaque année à une « énigme estudiantine » dont les composantes sont de deux ordres. Le premier élément à découvrir est le niveau de connaissance moyenne de la langue française. Il est d'une importance capitale, car la compétence linguistique conditionne la réception des textes littéraires français : si elle est déficitaire, la lecture des ouvrages classiques devient pénible, voire impossible. La Pologne faisant désormais partie de l'Union européenne, un phénomène inexistant auparavant a également fait son apparition, à savoir l'internationalisation des origines des étudiants. C'est ainsi qu'à côté des étudiants qui ont effectué leur scolarité secondaire en Pologne, il s'en trouve maintenant qui l'ont faite à l'étranger, parfois (mais pas nécessairement) dans un pays francophone. La connaissance du français des étudiants est ainsi extrêmement polarisée,

${ }^{14}$ D. Ulma, op. cit., p. 57.

${ }^{15}$ Ce sont les six semestres du premier cycle, au total 300 heures de cours environ (une heure de cours dure $45 \mathrm{~min}$.). 
avec aux deux extrêmes de parfaits bilingues et des candidats dont le niveau de français est totalement insuffisant. Le professeur est tenu d'évaluer le niveau moyen de connaissance du français dans sa classe et d'y adapter ses explications de texte (celles-ci n'étant évidemment pas possibles sans préparation lexicale et grammaticale), mais cette démarche a malgré tout fini par ne plus suffire.

Après l'arrivée à l'université des premiers bacheliers d'après la réforme, c'est-à-dire issus du lycée en trois ans, la détérioration constante de leur connaissance du français s'est révélée être un fait incontestable. La consternation des étudiants devant les textes qu'ils n'arrivaient pas à comprendre était si profonde, et tellement frustrante pour le professeur, qu'il est devenu nécessaire de changer de langue pour les cours magistraux et certains travaux dirigés de première année, de passer au polonais, et de leur permettre de lire les ouvrages intégraux obligatoires dans la traduction polonaise. Les extraits de textes français analysés en cours sont abordés en version originale (pour les textes médiévaux, ils sont doublés de traductions en français moderne), parfois accompagnée d'une traduction. Dans le prolongement de cette polonisation d'une partie des cours, et en suivant le principe d'accueillir un maximum de candidats, on a ouvert en 2011 une filière de formation destinée aux candidats sans connaissance préalable du français. L'innovation, appréhendée par une partie de corps professoral, s'est révélée très stimulante pour tous. Cette possibilité attire principalement des candidats qui envisagent les études françaises comme un complément. Tout en continuant leur première orientation d'études, souvent dans des domaines techniques, médicaux ou autres, ces jeunes entreprennent des études de français pour gagner un bagage en sciences humaines, pour satisfaire leur désir d'épanouissement culturel. Très motivés, appliqués, souvent passionnés pour la culture française, ils sont en outre capables de porter sur la littérature un regard « extérieur » rafraîchissant.

Le second élément que le professeur doit évaluer, ce sont les compétences littéraires des étudiants, ce que l'on nomme couramment leur bagage culturel. Cette vérification réserve des surprises, et exclut les présupposés. Avec la facilité de voyage à l'étranger, inexistante auparavant, et le développement de nouvelles technologies, l'horizon des possibilités d'approche de textes de toutes sortes, ouvrages anciens compris, de leurs gloses, des informations supplémentaires sur chaque domaine de la civilisation et de l'histoire, s'est élargi d'une manière autrefois inconcevable. Sylvaine Ahr reste sceptique quant à l'intérêt porté par les élèves français aux textes littéraires classiques, elle dénonce " le désintérêt quasi-général des adolescents, pour ces œuvres patrimoniales, jugées trop difficiles, trop longues à lire, et qui donnent une vision du monde trop éloignée de celui dans lequel ils évoluent $\rangle^{16}$. Cette attitude peut se retrouver chez une partie des lycéens polonais, mais il s'en trouve toujours qui manifestent un intérêt authentique pour la littérature savante. Les potentialités vertigineuses des bibliothèques

${ }^{16}$ S. Ahr, op. cit., p. 13. 
numériques, des films documentaires portant sur la littérature, l'histoire (politique, sociale, religieuse, de l'art) dont regorge l'internet, sont à portée de main de chaque élève, l'invitant à les explorer. Ce savoir, démystifié, concrétisé par l'image, peut pourtant devenir un piège pour les jeunes personnes qui ne possèdent pas les compétences de sélection nécessaires pour l'apprivoiser.

Dans cette situation, le professeur de littérature a perdu le privilège qu'il avait autrefois de détenir un savoir inaccessible. Il n'a cependant pas perdu son rôle de guide : au contraire, celui-ci est devenu crucial. Cette constatation m'amène à poser enfin la question essentielle : quelle est la place de la littérature française ancienne, médiévale ou classique, dans les études françaises ? Quel rôle peut-elle jouer dans la formation des jeunes romanistes? Les débats et les questionnements des scientifiques français, sur la place que la littérature doit occuper dans l'enseignement, montrent la complexité de l'approche des textes anciens ${ }^{17}$.

En entrant en contact avec la littérature française médiévale ou des siècles suivants, dans toute sa complexité, l'étudiant polonais reçoit de son professeur des indices concernant l'esthétique du texte, mais aussi des informations d'ordre social, culturel et historique qui lui permettent d'éviter les anachronismes d'interprétation. Il subit ainsi un processus d'acculturation et c'est là un premier objectif des études littéraires : on apprend la civilisation d'un pays étranger à travers sa littérature, ses grands textes classiques. Néanmoins, les lectures littéraires ne consistent pas à amasser une connaissance du contenu des textes : leur vraie fonction, moins mise en relief mais essentielle, est d'éveiller les esprits ${ }^{18}$. Les études de philologie sont un lieu privilégié pour s'épanouir, pour se forger un esprit critique. Cet épanouissement ne se réalise pas en amassant un savoir encyclopédique, qui est du reste facile à trouver en dehors des cours universitaires. Les textes anciens choisis avec rigueur et discernement par le professeur, même limités à des extraits, sont capables par leur altérité, doublée d'une similitude des idées, de mettre à l'épreuve les habitudes intellectuelles, de bouleverser la routine de pensée des étudiants plus qu'aucun autre ouvrage. Contrairement à la littérature française moderne, qui n'offre pas encore de recul suffisant pour une vision globale des grandes tendances esthétiques et thématiques et qui ne diffère pas foncièrement de son homologue polonaise, les textes anciens se prêtent à des synthèses multiples et, surtout, mettent en évidence un élément à la fois de permanence et de subjectivité des faits culturels. La littérature française ancienne est, pour l'étudiant polonais moyen, transgressive par excellence.

${ }^{17}$ Cf. S. Menant, «Littérature et enseignement : la réduction polymathique », R.H.L.F. 3, 2002, pp. 355-364.

18 Je développe cette idée dans M. Pawłowska, «L'enseignement de la littérature classique française en Pologne - quelques observations ", [dans :] Études françaises dans la société du XXI ${ }^{e}$ siècle : Défis et perspectives, E. Biardzka (dir.), Oficyna Wydawnicza LEKSEM, Łask 2011, pp. 83-90. 
Les jeunes sortent des lycées avec certains présupposés concernant les classements et les critères esthétiques des époques littéraires révolues. Ils sont aussi habitués aux explications uniques des œuvres et aux réponses autoritaires aux questions que pose un texte littéraire. En outre, ils sont entraînés à un enseignement thématique, donc chronologiquement éparpillé. La confrontation aux tendances générales de l'évolution esthétique leur montre les aspects inattendus, insoupçonnés, des ouvrages composés par de lointains auteurs, mais aussi la relativité des jugements esthétiques, parfois leur précarité, toujours le caractère arbitraire des classements et des hiérarchies. Certes, les étudiants s'attendent à découvrir pendant leurs études françaises un corpus de textes qui diffèrent par leur forme ou leur contenu de leur patrimoine littéraire, cependant, en règle générale, ils n'envisagent pas d'être mis en présence d'une altérité globale, combinant à la fois des éléments littéraires, socio-politiques et idéologiques. La littérature française médiévale qui commence quatre siècles avant les premiers textes écrits en polonais, dont les genres n'ont pas leurs répondants polonais, la doctrine du classicisme français qui prescrit des règles de la création poétique et qui reproduit en écho les idées de l'absolutisme, déroutent, mais aussi éveillent l'intérêt des étudiants polonais. Les décalages chronologiques entre les époques littéraires françaises et polonaises, qui ne deviennent compatibles qu'au XIX ${ }^{\mathrm{e}}$ siècle avec le courant romantique, perturbent les jeunes et les forcent à remettre en question leurs préjugés acquis ${ }^{19}$. Les textes anciens, présentés dans leur contexte esthétique et socio-historique, avec un support audiovisuel, sortent de l'anonymat de l'écrit et appellent à l'abandon des idées reçues. Les mots et les idées, appuyés par l'image, doublent l'impact de la portée du texte, dérangent, forcent les étudiants à reconsidérer leur vision simpliste du monde. Loin d'être une étude gratuite, la littérature ancienne constitue, en première année de philologie romane, une sorte de bélier médiéval, qui enfonce les portes de la forteresse où se réfugient les habitudes mentales, les présupposés, les stéréotypes. Elle est un outil de premier plan de l'ouverture d'esprit, de l'épanouissement des étudiants.

\section{TEACHING PRE-MODERN FRENCH LITERATURE: MEMORIES AND TESTIMONIES}

Summary

In Poland the collapse of the communist regime in 1989 had multiple and diverse implications for all the aspects of the life of Polish people. Its impact on the essential elements of the educational process, which are the student, the teacher and the curriculum, is unquestionable. The present

${ }^{19}$ Du type: « la littérature médiévale ne montre que le côté religieux de l'existence humaine », ou « la littérature baroque est omniprésente pendant tout le dix-septième siècle ». 
inquiry is structured around these three elements, in order to, firstly, foreground their complexities and, secondly, draw some conclusions regarding the place that should/can Pre-Modern French literature occupy in university curriculum in non-francophone countries.

Key words: educational process, Pre-Modern French literature, Polish students. 